\title{
RNF14 is a regulator of mitochondrial and immune function in muscle
}

\author{
Aaron B Ingham', Simone A Osborne ${ }^{1}$, Moira Menzies ${ }^{1}$, Suzie Briscoe', Wei Chen ${ }^{1}$, Kritaya Kongsuwan? \\ Antonio Reverter ${ }^{1}$, Angela Jeanes ${ }^{2}$, Brian P Dalrymple ${ }^{1}$, Gene Wijffels ${ }^{1}$, Robert Seymour ${ }^{1}$ and Nicholas J Hudson ${ }^{1 *}$
}

\begin{abstract}
Background: Muscle development and remodelling, mitochondrial physiology and inflammation are thought to be inter-related and to have implications for metabolism in both health and disease. However, our understanding of their molecular control is incomplete.

Results: In this study we have confirmed that the ring finger 14 protein (RNF14), a poorly understood transcriptional regulator, influences the expression of both mitochondrial and immune-related genes. The prediction was based on a combination of network connectivity and differential connectivity in cattle (a non-model organism) and mice data sets, with a focus on skeletal muscle. They assigned similar probability to mammalian RNF14 playing a regulatory role in mitochondrial and immune gene expression. To try and resolve this apparent ambiguity we performed a genome-wide microarray expression analysis on mouse C2C12 myoblasts transiently transfected with two Rnf14 transcript variants that encode 2 naturally occurring but different RNF14 protein isoforms. The effect of both constructs was significantly different to the control samples (untransfected cells and cells transfected with an empty vector). Cluster analyses revealed that transfection with the two Rnf14 constructs yielded discrete expression signatures from each other, but in both cases a substantial set of genes annotated as encoding proteins related to immune function were perturbed. These included cytokines and interferon regulatory factors. Additionally, transfection of the longer transcript variant 1 coordinately increased the expression of 12 (of the total 13) mitochondrial proteins encoded by the mitochondrial genome, 3 of which were significant in isolated pair-wise comparisons (Mt-coxll, Mt-nd2 and mt-nd4I). This apparent additional mitochondrial function may be attributable to the RWD protein domain that is present only in the longer RNF14 isoform.
\end{abstract}

Conclusions: RNF14 influences the expression of both mitochondrial and immune related genes in a skeletal muscle context, and has likely implications for the inter-relationship between bioenergetic status and inflammation.

Keywords: Mitochondria, Inflammation, RNF14, Muscle, Networks

\section{Background}

We are interested in understanding the regulation of muscle metabolism and its inter-relationships with development, exercise and ageing. A particular focus is the regulation of mitochondrial content which is reported to impact on metabolic syndrome in humans [1] and rats [2], feed efficiency in livestock [3] and mammalian ageing [4]. Mitochondrial content also contributes to athletic performance [5] and post-mortem meat quality [6] through

\footnotetext{
* Correspondence: nick.hudson@csiro.au

${ }^{1}$ CSIRO Animal, Food and Health Sciences, 306 Carmody Road, St. Lucia, Queensland, Australia

Full list of author information is available at the end of the article
}

the connection to muscle fibre type. The physiological connection between inflammation and muscle biology in the context of training, muscle remodelling and ageing is also of interest. For example, during exercise, muscle is routinely subject to various stressors, such as mechanical damage, hypoxia and $\mathrm{pH}$ decline that set in motion a proinflammatory cascade [7-9] which has implications for tissue remodelling. Previously, using a network science approach in cattle [10] we identified the product of the ring finger protein (RNF14) gene (aliases ARA54, TRIAD2), an incompletely characterised transcriptional regulator, as a factor that might influence not only mitochondrial transcription and function but also immune function. In this study we aimed to explore and validate the accuracy of

\section{Biomed Central}


this reverse-engineering under the tightly controlled experimental conditions afforded by in vitro cell culture.

A major foundation of our predicted function of the RNF14 protein was a bovine co-expression network [10]. Various metabolic and developmental processes were prioritised for further scrutiny on the basis of forming cohesive co-expression network gene sets or 'modules.' To build the network, bovine muscle sampled at different times during pre- and post-natal development, between genetically divergent breeds and following nutritional intervention, were subject to microarray analysis. By hunting in the module of interest for transcriptional regulators (DNA binding transcription factors and co-factors), or asking the related question "which transcriptional regulator has the highest absolute, average correlation to all the genes in the module?", we generated a ranked list of regulators predicted to control the processes in question.

These approaches correctly identified groups of genes already known to play a role in mammalian skeletal muscle biology, including master regulators of the cell cycle (E2F1), fast twitch muscle development (SIX1) and mitochondrial biogenesis $(E S R R A)$ [10]. Several regulatory molecules of unassigned or poorly documented function were also implicated in some of these processes, and they became candidates for future gene function validation efforts. While most genes are clearly defined within the network, one prominent gene, Ring Zinc Finger 14 (RNF14) a transcriptional co-activator known to bind the androgen receptor [11], gave apparently ambiguous results. Namely, it was assigned likely roles in two different processes by our analysis - immune function and mitochondrial function [10]. Given that the majority of connections in the co-expression network are positive, the prediction is that an increase in the activity of the 'regulator' will increase the expression of the 'target' genes.

A separate analysis on a different data set used a differential network strategy called Regulatory Impact Factor analysis (RIF) [12] to contrast mitochondrial-rich brown fat versus mitochondrial-poor white fat in mice. This analysis independently assigned high likelihood to a causal role for $R n f 14$ in driving the phenotype differences between these cell types, also suggestive of a role in mitochondrial function and content [13]. Little is known of the function of the RNF14 protein, other than it is broadly expressed across tissues [14] and is a transcriptional co-activator that interacts with the androgen receptor transcription factor in pathways relating to sex steroid signalling. From a structural perspective, there are six RNF14 transcript variants in humans and three in mouse, in both cases producing two different protein isoforms.

The objective of this study was to characterise the regulatory role of two RNF14 isoforms in mouse muscle.
We achieved this via experimental upregulation followed by functional analysis of the subsequent genome-wide transcriptional readout. We performed a transient transfection of transcript variants encoding the two different isoforms in mouse $\mathrm{C} 2 \mathrm{C} 12$ cells. The resultant gene expression perturbations in a number of chemokines, Interferon Regulatory Factors and related interferon signalling molecules support a role for Rnf14 in skeletal musclemediated immune and inflammatory function. Additionally, the longer transcript variant 1, which encodes a protein isoform containing an RWD domain, yielded a coordinate upregulation trend of all mitochondriallyencoded mitochondrial proteins present on the array platform (12 of the total 13) reinforcing the proposed link to the mitochondrion.

\section{Results}

\section{Expression constructs}

PCR resulted in two differently sized Rnf14 amplicons from mouse muscle cDNA. These amplicons were individually cloned and sequenced. In both cases the sequences exhibited $>99 \%$ sequence identity to the GenBank Rnf14 sequence. The longer sequence was $1457 \mathrm{bp}$ and BLASTN aligned this sequence to the Mus musculus Rnf14 transcript variant 1 . The shorter sequence we amplified was $1306 \mathrm{bp}$, and this was aligned by BLASTN to the Mus musculus Rnf14 transcript variant 3 (summarised in Figure 1).

Rnf14 transcript variant 1 contains an ORF capable of producing E3 ubiquitin protein ligase RNF14 protein isoform A and Rnf14 transcript variant 3 the ORF for E3 ubiquitin protein ligase RNF14 protein isoform B. The two isoforms are identical at the C-terminus end of the protein, while the longer isoforms has an RWD domain at the N-terminus end not present on the shorter isoform. Variant 2 does not encode a protein.

\section{Microarray expression measurements}

The array platform used to interrogate the $\mathrm{C} 2 \mathrm{C} 12$ response to transfection measures genome-wide transcriptional changes using 18,129 probes. This platform contains three probes predicted to bind Rnf14; ILMN_2675078, ILMN_2682811, ILMN_2868579. Their proposed binding sites are illustrated on Figure 1. Probe ILMN_2675078 was elevated $\sim 3.4$-fold in the variant 3 transfected cells and 1.1-fold in the variant 1 transfected cells. This 3.4-fold change made Rnf14 the 9th most differentially expressed (DE) gene found in cells transfected with variant 3 out of the 18,129 probes with detectable signals in at least one treatment. Correcting for an overall transfection efficiency of $\sim 10 \%$ implies that an individual transfected cell showed an increase in expression of 34-fold and 11-fold respectively.

The other two Rnf14 probes did not report a change in expression of $R n f 14$ following transfection, including 


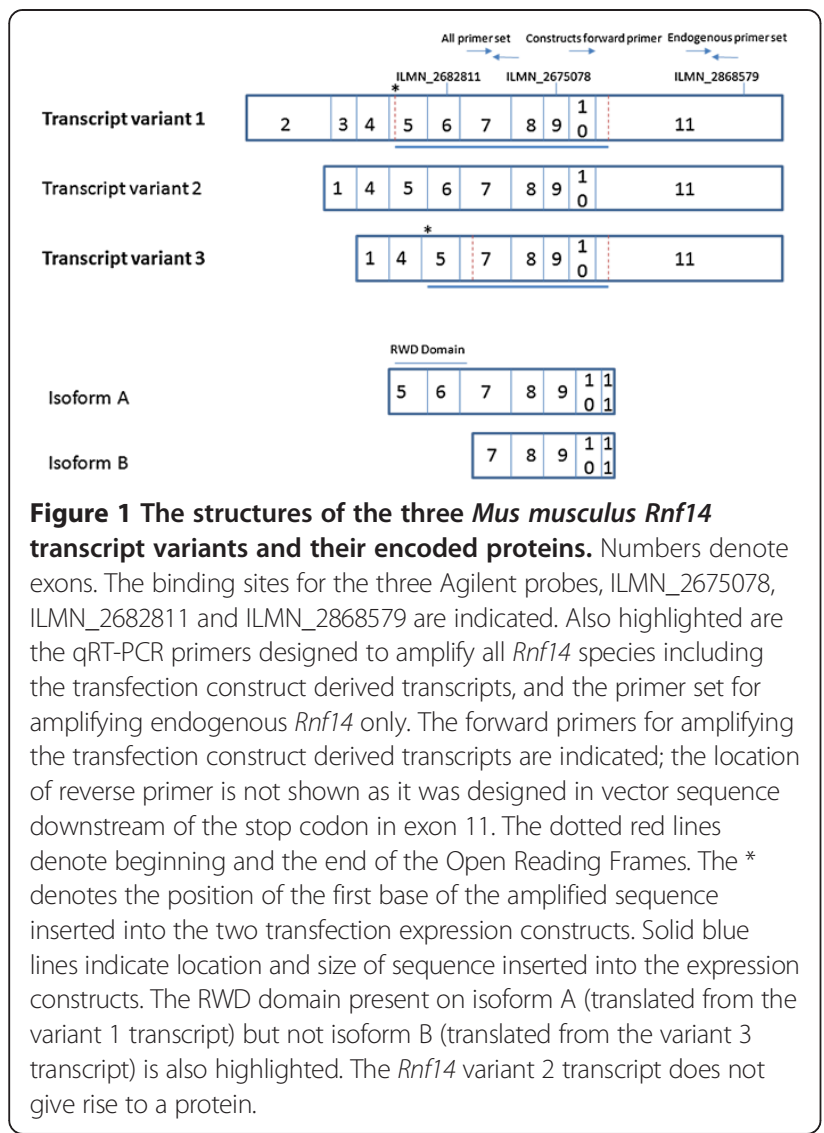

ILMN_2682811, predicted to bind variants 1 and 2 but not transcript variant 3 (Table 1). It is not clear whether these 2 probes are reporting correctly. To unravel these observations and to further document the technical implentation of the Rnf14 transfections we performed qRT-PCR on the RNA prepared from the transfected $\mathrm{C} 2 \mathrm{C} 12$ cultures using primers designed to detect the transfection construct produced mRNA and endogenous forms of Rnf14 mRNA.

\section{qRT-PCR expression measurements}

To compare and contrast the Rnf14 transcript variants in the different treatment groups, we designed a set of discriminatory qRT-PCR primers (Table 2). The ANOVA model for the analysis of $\mathrm{Ct}$ values from the qPCR experiments accounted for $93.03 \%$ of the total variation. The two main effects (primer and treatment) and their interaction were all highly significant $(\mathrm{P}<0.0001)$.

The normalised expression results are summarised in Table 3. All primer sets yielded unique dissociation curves indicating the presence of Rnf14 transcripts in the anticipated samples. The no template controls yielded no product in all cases. The transfection of Rnf14 variant 3 was clearly evident in the variant 3 transfected cells but not the other $\mathrm{C} 2 \mathrm{C} 12$ cultures $(\mathrm{P}<0.0001)$. The transcriptional output of this expression construct was also clearly detectable in the primer set that amplifies all Rnf14 mRNA species (i.e. endogenous and construct-based) yielding a 30 -fold increase $(\mathrm{P}<0.0001)$. The disparity between this result and the array fold-change is presumably attributable to the enhanced sensitivity of qRT-PCR.

Similarly, the transfection of the Rnf14 variant 1 was also clearly detectable in the variant 1 transfected cells but not the other cell cultures $(\mathrm{P}<0.0001)$. The expression by the construct was not as effective as for variant 3 and was not reflected in increased amplification by the all Rnf14 species (endogenous plus construct based) primer set, presumably because it forms a much more modest proportion of all the Rnf14 mRNA species in the system. There was very little variability in native $R n f 14$ expression (i.e. all three endogenous variants summed) across the three treatment groups. Overall, these findings imply the global transcriptional readout in the transfected $\mathrm{C} 2 \mathrm{C} 12$ cells described by the microarray platform can be attributed to the impact of the transfection of the Rnf14 variants.

\section{Functional enrichment analyses}

With regard to the microarray analysis, a mixed-model normalisation procedure was applied to the raw intensity values, as previously described [15]. Clustering on columns discriminates the treatments based on global considerations of the gene expression patterns. The visualisation of the clustering analysis indicated that two of the six control samples were outliers and hence were removed for the subsequent DE analysis. Overall, the global gene expression patterns in the two control groups (no transfection and empty construct transfection) could not

Table 1 Altered expression of Rnf14 transcripts in the Rnf14 variant 1 and variant 3 transfected C2C12 cells as reported by the three Illumina Rnf14 probes

\begin{tabular}{|c|c|c|c|c|c|}
\hline Probe ID & $\begin{array}{l}\text { Variant } 3 \text { transfected } \\
(\log 2)\end{array}$ & $\begin{array}{l}\text { Variant } 1 \text { transfected } \\
(\log 2)\end{array}$ & $\begin{array}{l}\text { Control cells } \\
(\log 2)\end{array}$ & $\begin{array}{l}\text { Variant } 3 \text { transfection } \\
\text { fold-change in } R n f 14 \\
\text { expression }\end{array}$ & $\begin{array}{l}\text { Variant } 1 \text { transfection } \\
\text { fold-change in } R n f 14 \\
\text { expression }\end{array}$ \\
\hline ILMN_2675078 & $10.54 \pm 0.11$ & $9.02 \pm 0.10$ & $8.78 \pm 0.13$ & 3.36 & 1.17 \\
\hline ILMN_2682811 & $7.44 \pm 0.13$ & $7.65 \pm 0.13$ & $7.53 \pm 0.14$ & No change & No change \\
\hline ILMN_2868579 & $12.73 \pm 0.17$ & $12.72 \pm 0.17$ & $12.73 \pm 0.08$ & No change & No change \\
\hline
\end{tabular}

Assuming a transfection efficiency of $\sim 10 \%$, the fold-changes for the transfected cells are $\sim 33$-fold and 12-fold on a per cell basis (based on ILMN_2675078).

The location of the probes in the different Rnf14 transcripts is given in Figure 1. 
Table 2 qRT-PCR primers used to detect endogenous and transfection constructed transcribed Rnf14 mRNA species

\begin{tabular}{lll}
\hline Transcripts & $\mathbf{5}$ 'primer & $\mathbf{3}$ primer \\
\hline Rnf14 all endogenous and all transfection construct & ctcaactgtccagagccaca & catggtaccaccaggctctt \\
Rnf14 all endogenous only & gccccattgtgttctcaact & gagccatgatgctcttcaca \\
Rnf14 variant 1 transfection construct & aattgaggaggacgacgatg & aggaactgcttccttcacga \\
Rnf14 variant 3 transfection construct & aattgaggaggacgacgatg & cgtagaatcgagaccgagga \\
Actb & gtgggccgccctaggcaccag & ctctttgatgtcacgcacgatttc \\
\hline
\end{tabular}

Transciption from the Actb gene was used as a control. A schematic of the known mouse Rnf14 mRNA species is presented in Figure 1.

be discriminated from each other. Consequently, the gene expression values for the two control groups were combined to form a single control for the purposes of computing DE between controls and treatments. The Rnf14 variant 3 and 1 transfected cells were both clustered separately to the controls (more so) and separate to each other (less so).

We computed a list of DE genes (Tables 4 and 5) as previously described [16], performing the analysis at the probe level. To explore the genome-wide expression output for functional enrichment we determined statistically significant DE for each treatment contrast. We then submitted the DE lists versus a background list of all genes present on the array to the GOrilla webtool [17], which uses hypergeometric statistics to determine functional enrichments.

With regard to upregulation following over-expression of the shorter variant 3, "response to biotic stimulus", "chemokine activity" and "extracellular region" gave $P$ values of $8.44 \mathrm{E}^{-12}, 2.93 \mathrm{E}^{-7}$ and $7.4 \mathrm{E}^{-14}$ for the Process, Function and Component ontological levels respectively.

With regard to upregulation following over-expression of the longer variant 1 , the top functional enrichments were "immune response," chemokine activity" and "extracellular region" with hypergeometric P-values of $1.98 \mathrm{E}^{-9}$, $3.83 \mathrm{E}^{-6}$ and $2.06 \mathrm{E}^{-10}$.

Various extracellular region components were clearly among the most downregulated genes following transfection with both variants (Table 5). The enrichment was highly significant in both cases, but more significant in the transcript $3\left(P=3.69 \mathrm{E}^{-19}\right)$ compared to the transcript 1 transfected cells $\left(P=1.32 \mathrm{E}^{-14}\right)$.

Contrasting effects of the variant 1 transfection with that of the variant 3 transfection yielded similar functional enrichments of "response to biotic stimulus" $\left(3.94 \mathrm{E}^{-8}\right)$, signal transducer activity $\left(2.15 \mathrm{E}^{-4}\right)$ and extracellular region $\left(1.77 \mathrm{E}^{-5}\right)$. The identity of the perturbed genes is further illustrated on Figure 2 and tabulated on Tables 4 and 5 . The normalised mean expression results for the entire data set are in Additional file 1. Transfection with the empty construct could not be discriminated from untransfected cells, ruling out the possibility that these responses are an experimental artefact relating to the presence of the construct. The overall spread of the perturbed transcripts is greater (4-fold DE in both directions) in the variant 3 transfected cells, which may reflect the greater abundance of the variant 3 based construct.

We also computed a modified DE metric called Phenotypic Impact Factors (PIF) [12,13], a product of the average abundance of the gene and its DE. We have previously found that this accounts for the increased noise of the rarer transcripts and increases the sensitivity for detecting DE of the more abundant transcripts [12]. Figure 3 illustrates those genes either DE or awarded a high PIF score in at least one of the two transfections. Immune and mitochondrial genes are highlighted based on functional annotations performed by importing the list into the DAVID web tool [18]. Immune genes and nuclear-encoded mitochondrial genes are prominent among the DE genes but the direction of change is not consistent.

On the other hand, among the significant PIF transcripts in the cells transfected with the long transcript variant are three of the 13 mitochondrially-encoded mitochondrial proteins (Mt-coxII, $M t$-nd2 and $M t$-nd4l). Moreover, a deeper exploration shows that all the mitochondrially-encoded genes represented on the array (12 of the 13) display a coherent trend of upregulation

Table 3 Expression (Ct LSM) of each Rnf14 mRNA species and beta actin based on qRT-PCR of variant 1 and variant 3 transfected C2C12 cells

\begin{tabular}{lllllc}
\hline Treatments & $\begin{array}{l}\text { All } \mathbf{3} \text { endogenous } \\
\text { and both constructs }\end{array}$ & All $\mathbf{3}$ endogenous & Variant $\mathbf{3}$ construct & Variant $\mathbf{1}$ construct & B actin \\
\hline Control & 24.99 & 26.59 & 31.93 (no amplification) & 35.6 (no amplification) & 23.16 \\
Transfected variant 3 & 20.17 & 26.73 & 19.41 & 34.19 (no amplification) & 23.36 \\
Transfected variant 1 & 24.89 & 26.92 & 30.32 (no amplification) & 29.19 & 23.10 \\
\hline
\end{tabular}

The standard error is 0.447 in all cases because we performed a system-wide normalization. 'No amplification' denotes multiple dissociation peaks i.e. non-specific amplification. 


\begin{tabular}{|c|c|c|}
\hline Rnf14 variant 3 versus controls & Gene ontology & Fold change (Log2) \\
\hline Ifit3 & Interferon induced protein & 2.22 \\
\hline G1p2 & Interferon induced protein & 2.20 \\
\hline 2510004L01Rik (RSAD2) & Interferon inducible & 2.09 \\
\hline Oasl2 & 2'-5'-oligoadenylate synthetase activity & 1.97 \\
\hline 2310061N23Rik (Ifi27/2a) & Interferon inducible & 1.96 \\
\hline $\mathrm{Ccl} 5$ & Chemokine ligand 5 & 1.91 \\
\hline Al481100 (Irgm2) & Interferon induced GTPase & 1.84 \\
\hline Usp18 & A member of the deubiquitinating protease family of enzymes & 1.76 \\
\hline Rnf14 & $\begin{array}{l}\text { This protein interacts with androgen receptor (AR) and may function as a } \\
\text { coactivator that induces AR target gene expression }\end{array}$ & 1.75 \\
\hline Gbp4 & Induced by interferon and hydrolyzes GTP & 1.73 \\
\hline Rnf14 variant 1 versus controls & Gene ontology & \\
\hline Plac8 & Placenta-specific, little known & 2.07 \\
\hline G1p2 & Interferon induced protein & 1.99 \\
\hline Oasl2 & 2'-5'-oligoadenylate synthetase activity & 1.82 \\
\hline $\mathrm{Ccl} 5$ & Chemokine ligand 5 & 1.68 \\
\hline LOC223672 (Apol9a) & Apolipoprotein, little known & 1.65 \\
\hline 2510004L01Rik & Interferon inducible & 1.61 \\
\hline 2310016F22Rik (Apol9b) & Apolipoprotein, little known & 1.54 \\
\hline ifit3 & Interferon induced protein & 1.42 \\
\hline Gbp4 & Guanylate-binding proteins induced by interferon & 1.30 \\
\hline Oas/1 & 2'-5'-oligoadenylate synthetase activity & 1.21 \\
\hline
\end{tabular}

Genes may be represented by more than one probe. The controls are a combination of untransfected cells and cells transfected with an empty construct.

in the variant 1 transfected cells based on at least one probe (Figure 3; Table 6; Additional file 2). The standard error bars for Figure 3 were calculated using the standard curve method [19]. A subset of these mitochondrially encoded genes that are significantly DE as assayed in pairwise comparisons are also highlighted on Figure 2. The upregulation of mitochondrially-encoded mitochondrial proteins was not observed in the $R n f 14$ variant 3 transfected cells (Additional file 2). The full list of DE and differentially PIF genes can be found in Additional file 3 .

\section{Motif analysis and bioinformatics}

We next attempted to identify regulatory motifs that are conserved among the RNF14 responsive genes in an attempt to determine the cellular pathway linking Rnf14 upregulation to the observed mitochondrial and inflammatory output. Hunting for conserved Transcription Factor Binding Sites 1000 bp upstream of the DE genes using Whole Genome RVista for mouse, enriched for Hfh3 in both the variant $1\left(\mathrm{P}=9.29-\log _{10}\right)$ and variant 3 transfection experiments $\left(\mathrm{P}=6.57-\log _{10}\right)$.

We also undertook a combination of bioinformatic analyses and literature mining. A protein motif analysis of RNF14 within UNIPROTKB indicated the presence of 1) an N-terminal destruction box which could act as a recognition signal for ubiquitin proteosome degradation and 2) RING type zinc finger essential for interaction with UBE2E2.

\section{Discussion}

In an attempt to infer the functional role of the RNF14 protein, previously found to be co-expressed with mitochondrial and immune genes in developing bovine longissimus muscle, we transfected each of two Rnf14 transcript variants into a mouse myoblast cell line. Interestingly, and despite low expression levels of that transcript, transfection with transcript variant 1 culminated in a significant upregulation in the transcription of three of the 13 mitochondrially-encoded mitochondrial genes (i.e. $M t-n d 4 L, M t$-coxII and $M t-n d 2)$ [12]. Furthermore, while we only have microarray expression data for 12 of the 13 of these genes ( $M t$-coxI is missing) a deeper exploration shows that the remaining nine all display a modest but coherent trend of upregulation. The direction of this observation is consistent with the initial network connections being based on positive rather than negative co-expression values. While the fold-change is 


\begin{tabular}{|c|c|c|}
\hline Rnf14 variant 3 versus controls & Gene ontology & Fold change (Log2) \\
\hline$\overline{D c n}$ & The encoded protein is a small cellular/pericellular matrix proteoglycan & -1.96 \\
\hline Myl1 & This gene encodes a fast myosin alkali light chain & -1.76 \\
\hline Spon2 & Extracellular matrix protein & -1.63 \\
\hline Prelp & $\begin{array}{l}\text { The protein encoded by this gene is a leucine-rich repeat protein present in } \\
\text { connective tissue extracellular matrix }\end{array}$ & -1.57 \\
\hline Den & The encoded protein is a small cellular or pericellular matrix proteoglycan & -1.50 \\
\hline Mylk & $\begin{array}{l}\text { This gene, a muscle member of the immunoglobulin gene superfamily, encodes } \\
\text { myosin light chain kinase which is a calcium/calmodulin dependent enzyme }\end{array}$ & -1.24 \\
\hline $\operatorname{Tgm} 2$ & $\begin{array}{l}\text { Transglutaminases are enzymes that catalyze the crosslinking of proteins by } \\
\text { epsilon-gamma glutamyl lysine isopeptide bonds }\end{array}$ & -1.20 \\
\hline Tnnt1 & This protein is the slow skeletal troponin T subunit & -1.18 \\
\hline Htra3 & Proteolysis & -1.17 \\
\hline Mylpf & Fast skeletal muscle & -1.16 \\
\hline Rnf14 variant 1 versus controls & Gene ontology & \\
\hline$D c n$ & The encoded protein is a small cellular or pericellular matrix proteoglycan & -0.88 \\
\hline Den & The encoded protein is a small cellular or pericellular matrix proteoglycan & -0.75 \\
\hline Myl1 & This gene encodes a fast myosin alkali light chain & -0.67 \\
\hline Prelp & $\begin{array}{l}\text { The protein encoded by this gene is a leucine-rich repeat protein present } \\
\text { in connective tissue extracellular matrix }\end{array}$ & -0.67 \\
\hline Spon2 & Extracellular matrix, innate immune response & -0.62 \\
\hline Col6a2 & Collagens are extracellular matrix proteins and have a triple-helical domain & -0.60 \\
\hline Enpp 1 & The encoded protein is a type II transmembrane glycoprotein & -0.57 \\
\hline 1190002H23Rik (Rgc32) & Cell cycle & -0.55 \\
\hline$|s| r$ & Immunoglobulin superfamily containing leucine-rich repeat & -0.54 \\
\hline Clatnf3 (Cors) & Beta oxidation, peroxisomal metabolism & -0.53 \\
\hline
\end{tabular}

Genes may be represented by more than one probe. The controls are a combination of untransfected cells and cells transfected with an empty construct.

only 1.1 to 1.4 -fold, all these transcripts are very abundantly expressed which provides a favourable signal to noise ratio for reliable detection. The array does not report on the 22 mitochondrially encoded tRNAs and two ribosomal RNAs that make up the remaining transcriptional output of the mammalian mitochondrion, which encodes 37 different genes in total.

The expression of a number of nuclear-encoded mitochondrial proteins was also upregulated following transfection of Rnf14 variant 1 (Figure 2). For example, Cmpk2 (alias Tyki) (2.4-fold up regulated) is a nucleoside monophosphate kinase that localises to the mitochondria and has previously been found to be tightly correlated with macrophage activation and inflammation [20]. A very recent publication has documented $R n f 14$ as a positive regulator of canonical Wnt signalling in human cells [21], with canonical Wnt signalling previously reported to be a potent activator of mitochondrial biogenesis [22]. This recent body of work clearly complements our findings linking Rnf14 with mitochondrial physiology.

While the broad transcriptional impact of Rnf14 variant 1 transfection on our samples was in line with our functional prediction, there were some interesting deviations. Firstly, the nuclear and mitochondrially-encoded mitochondrial proteins occupy distinct parts of the original bovine muscle co-expression network [10]. While Rnf14 sits in the nuclear-encoded portion of the in vivo bovine network, transfection with variant 1 appears to exert the most coherent transcriptional influence on the mitochondrially-encoded mitochondrial proteins. By way of contrast, Rnf14 variant 3 transfection did not lead to a detectable change on the expression of mitochondriallyencoded mitochondrial genes, despite an overabundance of the variant 3 transcript in the variant 3 transfected cells.

Both Rnf14 variants influenced expression of genes encoding proteins relating to immune function. Unlike the mitochondrially-encoded mitochondrial proteins upregulated observed in Rnf14 variant 1 transfected cells, genes belonging to inflammatory processes were both up- and downregulated. Prominent among the perturbed immune genes were chemokines (e.g. Ccl2, Ccl4, Ccl5, Ccl7, Cxcl10, Cxcl12), interferon regulatory factors and related interferon responsive and signalling genes (e.g. 

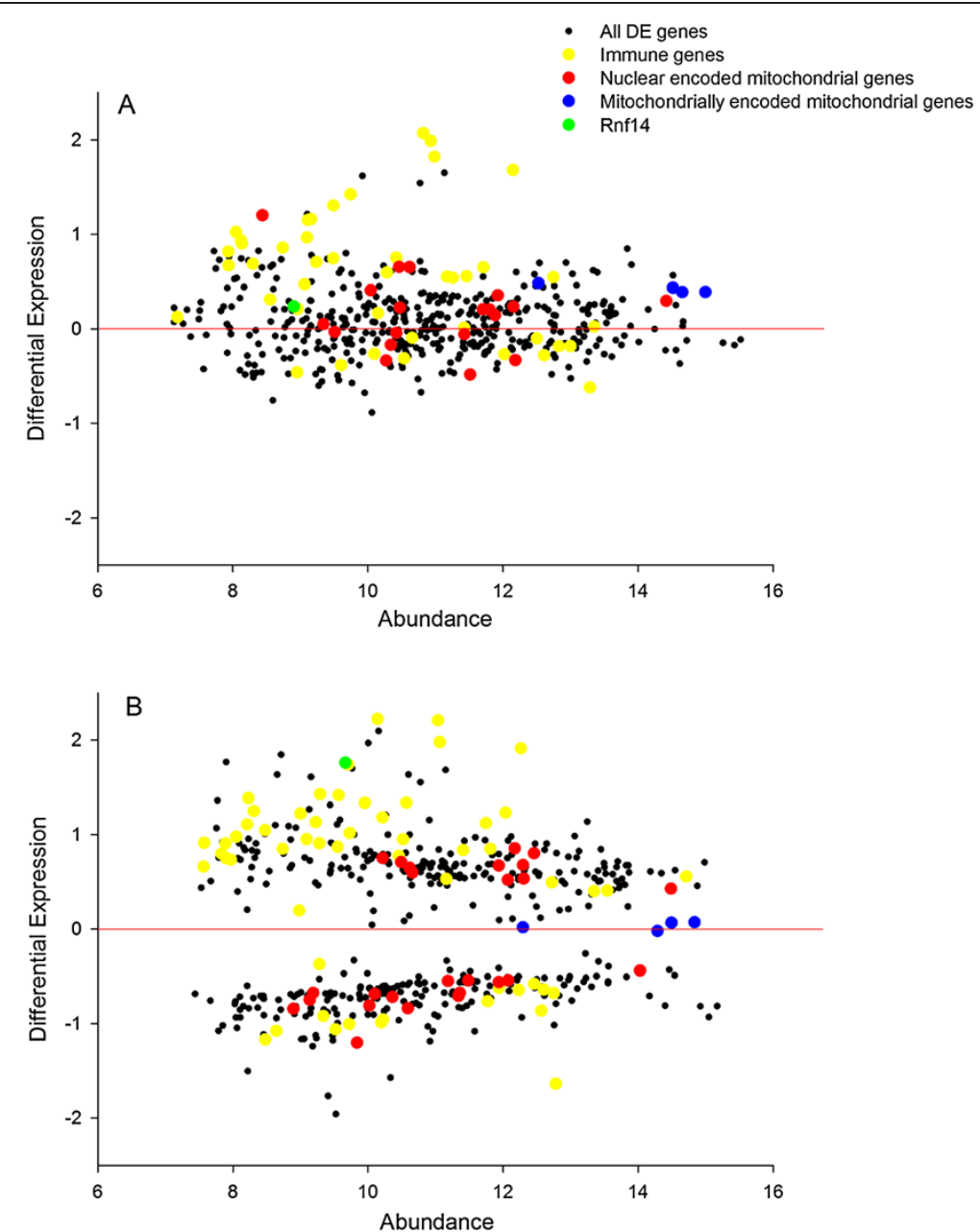

Figure 2 The effect of transfection of the Rnf14 variant 1 and 3 transcripts on genome-wide gene expression in C2C12 cells. Panel A Transfection of the Rnf14 transcript variant 1 on genome-wide gene expression supports a functional role in mitochondrial and immune function. The significant upregulation of several mitochondrially encoded mitochondrial proteins is noteworthy. Panel B Transfection of Rnf14 transcript variant 3 on genome-wide gene expression supports a functional role in immune function. The Rnf14 probe reporting these data is ILMN_2675078.

Irf1, Irf7, Irf9, Isg15, Isg20, Ifit2, Ifit3, Psmb8, Usp18, Adar, Gbp2). In humans following eccentric exercise, the in vivo inflammatory response includes activation of chemokines [23]. A number of these genes also imply apoptosis, a mitochondrial phenomenon [24]. These data go some way towards resolving the question posed by our apparently ambiguous (i.e. strong co-expression to both mitochondrial and immune genes) observations from the bovine muscle co-expression network, and imply that both mitochondrial and immune predictions are supported, depending on the particular transcript variant under consideration.

Both RNF14 motifs (N-terminal destruction box and RING type zinc finger) indicate some involvement in ubiquitin mediated proteolysis which ties in with apoptosis, and UBE2E2 is known to play a specific role in adaptive immunity signalling. The motif analysis shows that most of the large transcription factor motifs (Zinc fingers and RNA binding domains) of the protein reside in the $\mathrm{C}$-terminus shared by both isoforms, while the missing amino acids in the shorter isoform result in the loss of an RWD domain. We hypothesise that the RWD domain accounts for the mitochondrial response observed after transfection with Rnf14 transcript variant 1 . Recent work has emphasised deep functional connections between mitochondria and innate immunity in general $[25,26]$, and mitochondria and antiviral processing in particular [27], which is clearly of interest given the very same dual roles outlined here for RNF14.

The downregulation of a set of extracellular region and extracellular matrix transcripts following transfection with both Rnf14 transcript variants was unexpected. Example downregulated molecules common to both transfections included multiple collagen isoforms (Col14a1, Col6a2, Col6a1, Col8a2, Col16a1), other matrix structural components (Dcn, Mglap) and matrix remodelers (Mmp2, 


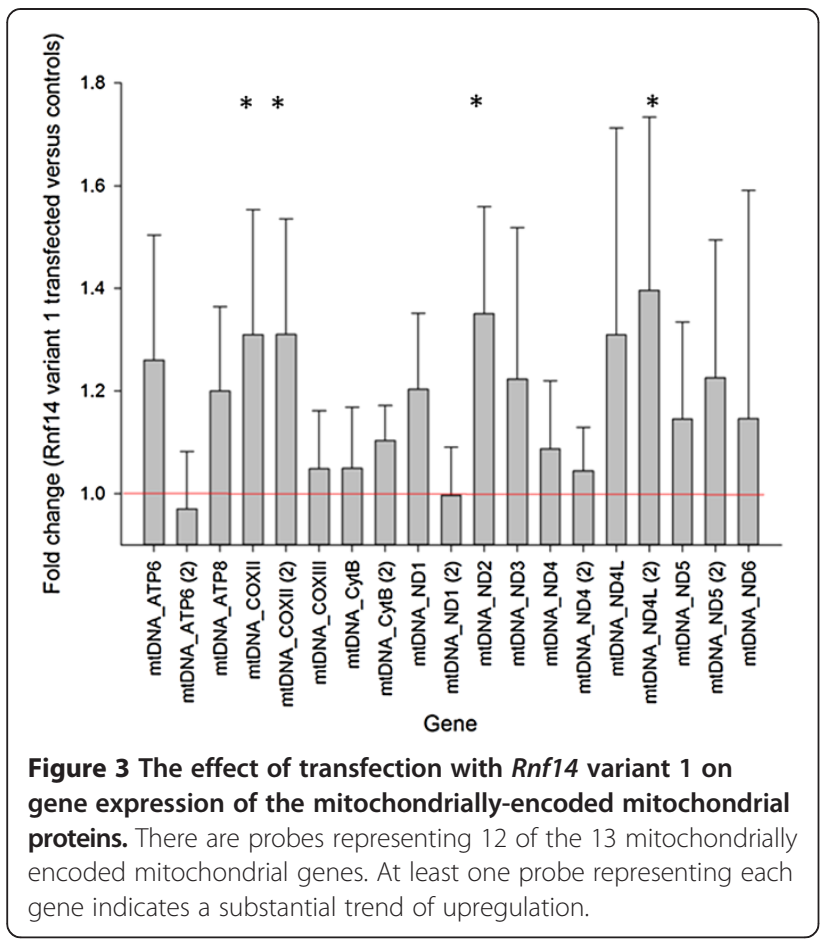

Adamts2). We ascribe these observations to one of two phenomena. On the one hand, it may reinforce the transmission of the immune signals we have observed, as it has been documented that the extracellular matrix plays a crucial role in the inflammatory process [28]. Alternatively, the signal may correspond to differences in myocyte progression through proliferation and differentiation, the transition through which is known to be accompanied by various changes in matrix-mediated adhesion [29].

Interpreting the various lines of evidence linking RNF14 protein to immune and mitochondrial functions is complicated by the cross-species sources of data. The original co-expression prediction of RNF14's gene function was made mainly from bovine expression data. Disentangling the various pieces of information is challenging given cattle are a non-model organism and we have incomplete knowledge of bovine functional genomics. For example, it is not clear how many bovine RNF14 transcript variants exist in total, which clearly complicates our (co-expression) interpretation of the Agilent probe that provided the original foundation for some of the predictions.

Nevertheless, the outcome of this validation experiment supports three of our systems biology gene discovery approaches: 1) Partial Correlation and Information Theory (PCIT) [30] 2) Module-to-Regulator analysis [10] and 3) Regulatory Impact Factors [12,13]. While there is some overlap in the exact molecules present in the coexpression modules and those perturbed in this subsequent transfection experiment (Prsmb8 and Irf1 being common to both in an immune context), the overlap is
Table 6 The absolute expression levels of the mitochondrially-encoded mitochondrial proteins in the Rnf14 variant 1 transfected cells versus controls

\begin{tabular}{lll}
\hline Gene & $\begin{array}{l}\text { Normalised mean } \\
\text { expression log 2 } \\
\text { (transfected variant 1) }\end{array}$ & $\begin{array}{l}\text { Normalised mean } \\
\text { expression log 2 } \\
\text { (controls) }\end{array}$ \\
\hline Mt-coxl/* & $15.19 \pm 0.09$ & $14.80 \pm 0.20$ \\
Mt-coxl/* & $14.84 \pm 0.09$ & $14.45 \pm 0.21$ \\
Mt-coxIII & $15.78 \pm 0.10$ & $15.71 \pm 0.03$ \\
Mt-atp6 & $14.76 \pm 0.04$ & $14.42 \pm 0.23$ \\
Mt-atp6 & $15.75 \pm 0.10$ & $15.79 \pm 0.03$ \\
Mt-atp8 & $15.48 \pm 0.05$ & $15.21 \pm 0.15$ \\
Mt-cytB & $15.66 \pm 0.08$ & $15.59 \pm 0.08$ \\
Mt-cytB & $15.87 \pm 0.05$ & $15.73 \pm 0.04$ \\
Mt-nd1 & $15.51 \pm 0.06$ & $15.24 \pm 0.13$ \\
Mt-nd1 & $15.93 \pm 0.09$ & $15.93 \pm 0.01$ \\
Mt-nd2* & $14.73 \pm 0.06$ & $14.29 \pm 0.19$ \\
Mt-nd3 & $8.45 \pm 0.17$ & $8.16 \pm 0.23$ \\
Mt-nd4 & $15.90 \pm 0.06$ & $15.84 \pm 0.05$ \\
Mt-nd4 & $15.66 \pm 0.07$ & $15.54 \pm 0.10$ \\
Mt-nd4/* & $12.76 \pm 0.16$ & $12.28 \pm 0.29$ \\
Mt-nd41 & $11.37 \pm 0.13$ & $10.98 \pm 0.37$ \\
Mt-nd5 & $12.52 \pm 0.05$ & $12.23 \pm 0.25$ \\
Mt-nd6 & $7.92 \pm 0.37$ & $7.72 \pm 0.23$ \\
Mt-nd5 & $7.94 \pm 0.07$ & $7.74 \pm 0.17$ \\
\hline
\end{tabular}

*Denotes pairwise significant differences at the $P<0.01$ level.

very patchy. This implies that predictions based on considerations of co-expression or differential co-expression are perhaps best made in terms of broad function rather than specific molecules.

\section{Conclusions}

Rnf14, which encodes a transcriptional co-factor, acts via two differentially spliced transcripts to modulate innate immune responses, tissue energy homeostasis and the tissue matrix. We do not know under what stimuli either transcript is produced by cells of different lineages. We have shown that gene expression information generated in a non-model organism can be used to develop hypotheses that can be validated in more conventional systems. The validation of this approach enhances the intrinsic value of many existing datasets generated in a range of species as it provides a methodology for detailed analysis of fundamental biological phenomena, such as mitochondrial transcription and biogenesis. Future work could aim to tease out whether one or more of the RNF14 protein isoforms are mitochondrially colocalised in addition to the mRNA transcripts being mitochondrially co-expressed, and/or whether in vivo evidence can be detected for a role in immune function 
and mitochondrial transcription or biogenesis to supplement the in vitro data we have presented.

\section{Methods}

\section{Prioritisation of RNF14 for experimental analysis}

Three separate lines of evidence were used to make a prediction of RNF14's possible role in immune versus mitochondrial function.

Firstly, a co-expression network on developing bovine muscle samples - inferred using the PCIT algorithm connected RNF14 to a dense nuclear-encoded mitochondrial module (comprising the following with correlations $>0.85$ MDH1, PDHA1, NDUFS2, ES1, UQCRC1, NDUFV1, MDH2, GOT2, NDUFA9, SOD2, CYCS, NDUFV2, SDHA, BRP44, ACO2, APOO) [10]. The position of RNF14 in the mitochondrial module of the co-expression network made a prediction about a role in mitochondrial function.

Secondly, a related co-expression based approach called Module-to-Regulator Analysis performed on the same data set showed that - in addition to its relationship to nuclear and mitochondrially-encoded genes - RNF14 also had very high overall co-expression patterns to the members of a separate immune module (IKZF3, PSMB9, MYO1G, PSMB8, A_73_104252, GIMAP7, A_73_109561, A_73_111087, CD48, A_73_110856, ASPA, IRF1, PTPN7, BOLA-DRA, A_73_116074, CD3G, BOLA-DRB3 and GIMAP6) [10] (Table 7).

Thirdly, application of the Regulatory Impact Factor (RIF) algorithm to an entirely independent data set (comparing high-mitochondrial content brown fat versus low-mitochondrial content white fat) awarded high likelihood of phenotype causality to the RNF14 protein [13].

\section{Amplification, cloning and transformation of two transcript variants of RNF14}

The mouse ortholog for RNF14 (GenBank accession NM_020012.1) was identified through sequence alignment to the bovine RNF14 protein. Mouse muscle cDNA was used as template for a PCR designed to amplify the full length mRNA encoding the Rnf14 gene. To increase recognition of the first ATG by the ribosome, the forward primer incorporated the mammalian Kozak sequence (CCATGG) prior to the start of the Open Reading Frame. PCR primers also incorporated vector sequence, restriction enzyme sites used for cloning ( $\mathrm{NcOI}$ at 5' and XhoI 3') and gene specific sequence. The forward primer for PCR was 5'- GAGATATGCCACCATGGAGT CGGCAGAAGACCTGGAAGCCCAG-3' and the reverse primer 5'- GTGATGGTGGTGCTCGAGGTCGTCGTCG TCGTCTTCATCATCGT-3'.

A conventional PCR with a $50^{\circ} \mathrm{C}$ annealing temperature using high fidelity Taq polymerase, amplified a band of 1457 base pairs. This was gel purified and cloned into the pTANDEM-1 vector (EMD4 Biosciences, Merck, USA) using a fusion homologous recombination method. The resultant expression plasmid contained a $\mathrm{His}_{6}$ tag at the $\mathrm{C}$ terminus of the RNF14 gene for protein purification and detection by antibodies.

In a separate PCR reaction a shorter Rnf14 transcript variant was also amplified (1306 bp), using the forward primer 5'-ATGTCGGCAGAAGACCTGGAAGC-3' and the reverse primer 5'-CTAGTCGTCGTCGTCGTCTT CATCATCG-3'. This PCR product was cloned into the pcDNA3.1/V5-his TOPO TA vector (Invitrogen, USA).

The two variants are referred to as Rnf14 transcript variant 1 and Rnf14 transcript variant 3 . Following

Table 7 Top 10 transcriptional regulators showing the highest absolute, average co-expression to all the genes in the bovine muscle immune module

\begin{tabular}{|c|c|}
\hline $\begin{array}{l}\text { Transcriptional regulator } \\
\text { (average correlation to immune module) }\end{array}$ & Gene ontology \\
\hline IRF1 (0.905) & $\begin{array}{l}\text { IRF1 encodes interferon regulatory factor } 1 \text {, a member of the interferon regulatory transcription factor } \\
\text { (IRF) family. IRF1 also functions as a transcription activator of genes induced by interferons a, } \beta \text { and } \gamma \text {. } \\
\text { Further, IRF1 has been shown to play roles in regulating apoptosis }\end{array}$ \\
\hline RNF14 (0.901) & $\begin{array}{l}\text { This protein interacts with androgen receptor (AR) and may function as a co-activator that induces } A R \\
\text { target gene expression }\end{array}$ \\
\hline TEAD1 (0.872) & $\begin{array}{l}\text { This gene encodes a ubiquitous transcriptional enhancer factor that is a member of the TEA/ATTS } \\
\text { domain family }\end{array}$ \\
\hline LRRFIP1 (0.849) & Phosphorylated in response to immunologic stimuli \\
\hline EPAS1 (0.845) & Myoblast cell fate commitment \\
\hline NR1D2 (0.844) & Nuclear Hormone Receptor involved in carbohydrate and lipid metabolism \\
\hline $\operatorname{RORC}(0.841)$ & Regulates IL2 expression. IL2 is an inflammatory cytokine \\
\hline PCGF5 (0.840) & Little known \\
\hline PHF12 (0.838) & Little known \\
\hline HOXD8 (0.838) & $\begin{array}{l}\text { The homeobox genes encode a highly conserved family of transcription factors that play an important } \\
\text { role in morphogenesis }\end{array}$ \\
\hline
\end{tabular}


transformation into TOP10 chemically competent E.coli, clones were picked and diagnosed for the presence of the insert in the correct orientation using restriction digests (XhoI and NcoI) followed by gel electrophoresis. Clones predicted to contain the correct insert were sequenced from both ends of the expression construct using the BGH, T7 and pTandem1 Tandem DOWN1 primer and aligned to the GenBank mouse RNF14 sequence.

Expression constructs containing the correct sequence were midi-prepped (Qiagen) following the manufacturer's instructions and stored at $1 \mu \mathrm{g} / \mu \mathrm{l}$ at $-20^{\circ} \mathrm{C}$ in preparation for transfection. The Qiagen midi-prepping protocol removes LPS and provides transfection-ready reagents.

A control construct containing no insert (hereon referred to as "empty") was also produced and subsequently used for transfection. A separate control was derived from untransfected cells.

\section{Cell culture and transfection conditions}

The murine myoblast cell line, C2C12, was obtained from the American Type Culture Collection and cultured in DMEM supplemented with penicillin/streptomycin and $10 \%$ fetal bovine serum. Transfection conditions for the C2C12 cells (passage numbers 15 - 20) were explored for a range of confluences and Lipofectamine 2000 (Invitrogen) transfection reagent: DNA ratios. A pEGFP.C3 vector containing Green Fluorescent Protein (GFP) was used to visualise transfection efficiency under a Zeiss AX10 fluorescence microscope fitted with an Axiocam camera. As previously reviewed [31], we found that $\mathrm{C} 2 \mathrm{C} 12$ cells transfected with a low transfection efficiency of $\sim 10 \%$. Optimal cell number was found to be $5 \times 10^{4}$ cells/well transfected with $5 \mu \mathrm{l}$ of Lipofectamine 2000 and 800 ng DNA per well for 48 hours.

The experiment was run in two 24-well Geltrex-coated plates using the optimised procedure. Cells were cultured for 24 hours prior to transfection. At the time of transfection the cells were $\sim 50 \%$ confluent. Two independent controls were used, one with no transfection and the other through transfection of the empty construct.

Twelve independent wells were run for each of the four treatment groups (no transfection, empty construct, Rnf14 short transcript variant and Rnf14 long transcript variant) giving a 48 well cell culture experiment in total. At 48 hours post transfection the cells from each well were harvested for RNA, while a GFP transfection run in parallel was fixed and stained to provide an estimate of transfection efficiency.

\section{RNA extraction and microarray hybridisation}

Total RNA was independently extracted from each of the 48 cell culture wells using QIAshredder homogenates and
QIAgen RNeasy columns (Qiagen), incorporating the oncolumn DNase treatment (Ambion) to remove genomic DNA contamination. RNA integrity and purity was assayed visually by gel electrophoresis and additionally by $\mathrm{A}_{260 / 280}$ spectrophotometry. All the RNA samples resolved into discrete $16 \mathrm{~S}$ and $26 \mathrm{~S}$ ribosomal RNA bands.

Because each of the four treatment groups comprised 12 independent RNA samples, these 12 were pooled into 3 major groups of 4 replicates with each replicate contributing $0.5 \mu \mathrm{g}$. This process yielded 12 by $2.0 \mu \mathrm{g}$ pooled RNA samples as follows (no transfection 1, no transfection 2, no transfection 3, empty construct 1, empty construct 2 , empty construct 3 , short transcript 1 , short transcript 2, short transcript 3 , long transcript 1 , long transcript 2, long transcript 3).

The 12 RNA pools were submitted to the Gene Expression Centre at the University of Queensland's Institute for Molecular Bioscience for cDNA synthesis and hybridisation to the Illumina WG6 mouse microarray platform. This facility performed an additional set of quality checks based on possession of an RNA Integrity Number (RIN) $>8$ assayed on a Bioanalyzer.

\section{Statistical analysis}

The analytical procedures to normalize the data and to identify differentially expressed genes were based on methods developed by our group and published elsewhere. In particular, we followed the methodology described in $[15,32,33]$.

The raw data from the Illumina Microarray System contained expression signals from 45,281 probes across the 12 hybridizations.

\section{Initial normalization}

We base-2 Log-transformed the expression signals to stabilize the variance, capturing only those probes with a "detectable" signal $(\mathrm{P}<0.01)$ in at least one of the 12 hybridizations. This produced a "cleaned" dataset of 18,129 Illumina probes.

\section{Chip normalization}

The "cleaned" dataset was subject to a within chip normalization in which each signal was normalized by subtracting the within-chip mean and dividing by the within-chip standard deviation. The chips were therefore standardized to have a mean $(\mu)$ of 0 and a standard deviation $(\sigma)$ of 1 , enabling data from different chips to be combined without the risk of being influenced by differences in mean or SD. This allows subsequent linear functions to be more reasonably derived, in our case computation of differential expression. The resulting normalized dataset was subjected to hierarchical cluster analyses using the PermutMatrix software [34]. 


\section{Identification of DE/DPIF genes}

For the identification of DE/DPIF genes we focused on two contrasts: variant 1 versus controls and variant 3 versus controls. Following [35] a two-component normal mixture model was fitted to identify DE/DPIF genes. Parameters of the mixture model were estimated using the EMMIX-GENE software [36] and an estimated experimentwise false discovery rate (FDR) of $<1 \%$ used as the threshold for determining which genes are DE/DPIF.

\section{qRT-PCR}

We used Superscript III (Invitrogen) for cDNA synthesis for the 12 pools following the manufacturer's instructions, in each case using lug of the same RNA stock hybridised to the microarray platforms. The resultant 20 $\mathrm{ul}$ of neat cDNA was diluted to make a 1:10 solution for qPCR.

Four primer sets were designed to discriminate between endogenously produced and transfection construct generated Rnf14 mRNA species (Table 2). In each case, Primer3 software was used for primer design to amplify $100-200 \mathrm{bp}$ of sequence based on a $60^{\circ} \mathrm{C}$ annealing temperature. We accepted the remainder of Primer 3's default settings.

To assay expression all endogenous Rnf14 transcripts we designed primers in the 3' UTR of exon 11 (Figure 1). This exon is shared by all variants, but will not amplify construct based transcripts because they possess vector sequence downstream of the stop codon. To assay all Rnf14 transcripts (endogenous and from the transfection constructs) we designed primers in exons 7-8 that are shared by all the mRNA species. To assay transcription relating to the two expression constructs (forward primer visualised on Figure 1) we designed the forward primer prior to the stop codon in exon 11 and the reverse primer in the downstream vector sequence.

For the qRT-PCR assays we used a Sybr Green master mix with $900 \mathrm{nM}$ of each primer and a constant amount of cDNA run on an Applied Biosystems ViiA7 thermal cycler (Forster City, CA, USA) in $10 \mu$ reactions. qPCR reactions were run in triplicate with a no template control for each primer set. Expression was normalised using beta-actin as a reference gene. Because the empty construct and untransfected cells could not be discriminated by microarray, we used the empty construct as the control RNA's for qRT PCR.

\section{qRT PCR statistical analysis}

Following previously described approaches [37] statistical analysis was carried out using SAS software 9.2 (SAS Institute, Cary, NC), using triplicate Ct values per sample. In particular the $\mathrm{Ct}$ values were normalized by fitting an analysis of variance (ANOVA) model that accounted for "within primer across treatment" and "within treatment across primer" variation. A general linear model approach was used consisting of the following: Expression $(\mathrm{Ct}) \sim$ Primer + Treatment + Primer"Treatment + Error. Finally, for each Primer, the corresponding contrast of least-square means for $\mathrm{Ct}$ values in the Treatment of interest was used to ascertain differential expression.

\section{Additional files}

Additional file 1: The normalized mean expression values $(\log 2)$ for all genes across the 12 biological replicates.

Additional file 2: The fold changes in the mitochondrially-encoded mitochondrial proteins following Rnf14 transfection.

Additional file 3: Those genes significantly DE and DPIF following Rnf14 transfection.

Competing interests

The authors declare they have no competing interests.

Authors' contributions

$\mathrm{SAO}, \mathrm{SB}, \mathrm{WC}, \mathrm{KK}, \mathrm{AJ}, \mathrm{MM}$ and NJH performed the experiments. AR performed the statistical analyses. ABI, GW and RS helped design and interpret the experiments. BPD performed the bioinformatic analysis. NJH drafted the manuscript. RBS, GW and NJH resourced and managed the project.

All authors read, contributed to, and approved the manuscript.

\section{Acknowledgements}

NJH was in receipt of the Department of Agriculture, Fisheries and Forestry Young Scientist and Innovators Ministers' Award co-sponsored by Meat and Livestock Australia. The authors wish to thank Dr. Andrew Kotze for critical reading of an earlier draft of this manuscript.

\section{Author details}

${ }^{1}$ CSIRO Animal, Food and Health Sciences, 306 Carmody Road, St. Lucia, Queensland, Australia. ${ }^{2}$ School of Biomedical Sciences, University of Queensland, St. Lucia, Queensland, Australia.

Received: 30 April 2013 Accepted: 21 January 2014

Published: 29 January 2014

\section{References}

1. Holloway GP, Bonen A, Spriet LL: Regulation of skeletal muscle mitochondrial fatty acid metabolism in lean and obese individuals. Am J Clin Nutr 2009, 89(1):455S-462S.

2. Couturier A, Ringseis R, Mooren FC, Kruger K, Most E, Eder K: Carnitine supplementation to obese Zucker rats prevents obesity-induced type ॥ to type I muscle fiber transition and favors an oxidative phenotype of skeletal muscle. Nutr Metab (Lond) 2013, 10(1):48.

3. Hudson NJ: Symmorphosis and livestock bioenergetics: production animal muscle has low mitochondrial volume fractions. J Anim Physiol Anim Nutr (Berl) 2009, 93(1):1-6.

4. Short KR, Bigelow ML, Kahl J, Singh R, Coenen-Schimke J, Raghavakaimal S, Nair KS: Decline in skeletal muscle mitochondrial function with aging in humans. Proc Natl Acad Sci USA 2005, 102(15):5618-5623.

5. Karlsson J, Sjodin B, Tesch P, Larsson L: The significance of muscle fibre composition to human performance capacity. Scand J Rehabil Med Suppl 1978, 6:50-61.

6. Kim JM, Lee KT, Lim KS, Park EW, Lee YS, Hong KC: Effects of p.C430S polymorphism in the PPARGC1A gene on muscle fibre type composition and meat quality in Yorkshire pigs. Anim Genet 2010, 41(6):642-645.

7. Maclntyre DL, Sorichter S, Mair J, Berg A, McKenzie DC: Markers of inflammation and myofibrillar proteins following eccentric exercise in humans. Eur J Appl Physiol 2001, 84(3):180-186.

8. Malm C, Lenkei R, Sjodin B: Effects of eccentric exercise on the immune system in men. J Appl Physiol 1999, 86(2):461-468. 
9. Malm C, Nyberg P, Engstrom M, Sjodin B, Lenkei R, Ekblom B, Lundberg I: Immunological changes in human skeletal muscle and blood after eccentric exercise and multiple biopsies. J Physiol 2000, 529(Pt 1):243-262.

10. Hudson NJ, Reverter A, Wang Y, Greenwood PL, Dalrymple BP: Inferring the transcriptional landscape of bovine skeletal muscle by integrating co-expression networks. PLoS One 2009, 4(10):e7249.

11. Kang HY, Yeh S, Fujimoto N, Chang C: Cloning and characterization of human prostate coactivator ARA54, a novel protein that associates with the androgen receptor. J Biol Chem 1999, 274(13):8570-8576.

12. Hudson NJ, Reverter A, Dalrymple BP: A differential wiring analysis of expression data correctly identifies the gene containing the causal mutation. PLoS Comput Biol 2009, 5(5):e1000382.

13. Reverter A, Hudson NJ, Nagaraj SH, Perez-Enciso M, Dalrymple BP: Regulatory impact factors: unraveling the transcriptional regulation of complex traits from expression data. Bioinformatics 2010, 26(7):896-904.

14. Liu X, Yu X, Zack DJ, Zhu H, Qian J: TiGER: a database for tissue-specific gene expression and regulation. BMC Bioinf 2008, 9:271.

15. Reverter A, Barris W, McWilliam S, Byrne KA, Wang YH, Tan SH, Hudson N, Dalrymple BP: Validation of alternative methods of data normalization in gene co-expression studies. Bioinformatics 2005, 21(7):1112-1120.

16. Moser RJ, Reverter A, Kerr CA, Beh KJ, Lehnert SA: A mixed-model approach for the analysis of CDNA microarray gene expression data from extremeperforming pigs after infection with Actinobacillus pleuropneumoniae. J Anim Sci 2004, 82(5):1261-1271.

17. Eden E, Navon R, Steinfeld I, Lipson D, Yakhini Z: GOrilla: a tool for discovery and visualization of enriched $\mathrm{GO}$ terms in ranked gene lists. BMC Bioinf 2009, 10:48

18. da Huang W, Sherman BT, Lempicki RA: Systematic and integrative analysis of large gene lists using DAVID bioinformatics resources. Nat Protoc 2009, 4(1):44-57.

19. Livak KJ: Relative quantitation of gene expression. In User bulletin \#2 ABI prism 7700 sequence detection system. Applied Biosystems; 1997.

20. Xu Y, Johansson M, Karlsson A: Human UMP-CMP kinase 2, a novel nucleoside monophosphate kinase localized in mitochondria. J Biol Chem 2008, 283(3):1563-1571.

21. Wu B, Piloto S, Zeng W, Hoverter NP, Schilling TF, Waterman ML: Ring Finger Protein 14 is a new regulator of TCF/beta-catenin-mediated transcription and colon cancer cell survival. EMBO Rep 2013 14(4):347-355

22. Yoon JC, Ng A, Kim BH, Bianco A, Xavier RJ, Elledge SJ: Wnt signaling regulates mitochondrial physiology and insulin sensitivity. Genes Dev 2010, 24(14):1507-1518.

23. Chen YW, Hubal MJ, Hoffman EP, Thompson PD, Clarkson PM: Molecular responses of human muscle to eccentric exercise. J Appl Physiol 2003, 95(6):2485-2494

24. Chiarugi A, Moskowitz MA: Cell biology. PARP-1-a perpetrator of apoptotic cell death? Science 2002, 297(5579):200-201.

25. West AP, Shadel GS, Ghosh S: Mitochondria in innate immune responses Nat Rev Immunol 2011, 11(6):389-402

26. Khoo J, Nagley P, Mansell A: Mitochondria: an unexpected force in innate immunity. Aust Biochemist 2013, 44(1):17-20.

27. Scott I: The role of mitochondria in the mammalian antiviral defense system. Mitochondrion 2010, 10(4):316-320.

28. Sorokin L: The impact of the extracellular matrix on inflammation. Nat Rev Immunol 2010, 10(10):712-723.

29. Moran JL, Li Y, Hill AA, Mounts WM, Miller CP: Gene expression changes during mouse skeletal myoblast differentiation revealed by transcriptional profiling. Physiol Genomics 2002, 10(2):103-111.

30. Reverter A, Chan EK: Combining partial correlation and an information theory approach to the reversed engineering of gene co-expression networks. Bioinformatics 2008, 24(21):2491-2497.

31. Balci $B$, Dincer P: Efficient transfection of mouse-derived $\mathrm{C} 2 \mathrm{C} 12$ myoblasts using a matrigel basement membrane matrix. Biotechnol J 2009, 4(7):1042-1045.

32. Reverter A, Ingham A, Lehnert SA, Tan SH, Wang Y, Ratnakumar A, Dalrymple BP: Simultaneous identification of differential gene expression and connectivity in inflammation, adipogenesis and cancer. Bioinformatics 2006, 22(19):2396-2404.

33. Nagaraj SH, Reverter A: A Boolean-based systems biology approach to predict novel genes associated with cancer: Application to colorectal cancer. BMC Syst Biol 2011, 5:35.
34. Caraux G, Pinloche S: PermutMatrix: a graphical environment to arrange gene expression profiles in optimal linear order. Bioinformatics 2005, 21(7):1280-1281.

35. McLachlan GJ, Bean RW, Jones LB: A simple implementation of a normal mixture approach to differential gene expression in multiclass microarrays. Bioinformatics 2006, 22(13):1608-1615.

36. McLachlan GJ, Bean RW, Peel D: A mixture model-based approach to the clustering of microarray expression data. Bioinformatics 2002, 18(3):413-422.

37. De Jager N, Hudson NJ, Reverter A, Wang YH, Nagaraj SH, Café LM, Greenwood PL, Barnard RT, Kongsuwan KP, Dalrymple BP: Chronic exposure to anabolic steroids induces the muscle expression of oxytocin and a more than fiftyfold increase in circulating oxytocin in muscle. Physiol Genomics 2011, 43(9):467-478.

doi:10.1186/1752-0509-8-10

Cite this article as: Ingham et al.: RNF14 is a regulator of mitochondrial and immune function in muscle. BMC Systems Biology 2014 8:10.

\section{Submit your next manuscript to BioMed Central and take full advantage of:}

- Convenient online submission

- Thorough peer review

- No space constraints or color figure charges

- Immediate publication on acceptance

- Inclusion in PubMed, CAS, Scopus and Google Scholar

- Research which is freely available for redistribution

Submit your manuscript at www.biomedcentral.com/submit
C) Biomed Central 